\title{
Traduire
}

Une autre perspective sur r tr traduction

Revue française de la traduction

$241 \mid 2019$

La formation à I'honneur

\section{La plus-value de la biotraduction face à la machine}

Le nouveau défi des formations aux métiers de la traduction

\section{Rudy Loock}

\section{(2) OpenEdition}

Journals

Édition électronique

URL : http://journals.openedition.org/traduire/1848

DOI : $10.4000 /$ traduire.1848

ISSN : 2272-9992

Éditeur

Société française des traducteurs

Édition imprimée

Date de publication : 16 décembre 2019

Pagination : 54-65

ISSN : 0395-773X

Référence électronique

Rudy Loock, «La plus-value de la biotraduction face à la machine», Traduire [En ligne], 241 | 2019, mis en ligne le 16 décembre 2019, consulté le 05 février 2020. URL : http://journals.openedition.org/ traduire/1848; DOI : 10.4000/traduire.1848 


\section{La plus-value de la biotraduction face à la machine}

Le nouveau défi des formations aux métiers de la traduction.

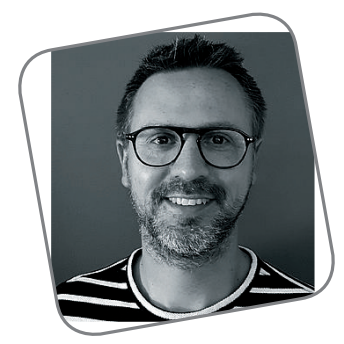

Rudy Loock

\section{Introduction}

Après l'informatisation importante des métiers de la traduction dans les années 1990 et 2000 , les formations universitaires ont en ce début de $x \times I^{e}$ siècle un nouveau défi majeur à relever. Avec la révolution numérique et les incontestables progrès de la traduction automatique/machine (TA) depuis l'arrivée de la TA neuronale, il semble incontournable d'enseigner aux futurs professionnels la façon dont ils peuvent et doivent dégager leur plus-value de traducteur humain (aussi appelé «biotraducteur $r^{1}$ ) par rapport à la machine et face à des discours parfois caricaturaux sur le sujet. Les progrès de la TA ne peuvent être ignorés: ils sont en train de bouleverser les méthodes de travail et le modèle économique 
du secteur. Ainsi, en 2018, la proportion d'entreprises de services linguistiques européennes déclarant avoir recours à la TA a dépassé pour la première fois les 50 \% (2018 Study of the European language ${ }^{2}$ ). L'utilisation de la TA au sein des logiciels de traduction assistée par ordinateur (par exemple SDL Trados Studio, memoQ), sous la forme de fonctionnalité intégrée ou de plug-in, se généralise. Parallèlement, l'activité ne ralentit pas: au niveau mondial, le secteur des services linguistiques continue de se développer, avec en 2019 une croissance de 6,62\% par rapport à 2018 (Common Sense Advisory, The Language Services Market: $2019^{3}$ ). II s'agit donc certes d'une technologie "disruptive» pour reprendre un terme à la mode, mais qui ne semble pas devoir remplacer le traducteur humain, contrairement à ce que l'on entend parfois. Les changements sont certains, et le secteur des métiers de la traduction, comme moult autres secteurs d'activité, est concerné par les progrès de l'intelligence artificielle. Pour les formations universitaires, l'heure est donc à l'adaptation. II s'agit de préparer les étudiants aux futures réalités du marché. Et même si celles-ci sont en partie inconnues, il incombe aux formateurs de faire prendre conscience aux futurs professionnels de ce que la machine peut faire et ne peut pas faire, afin de "démystifier» (terme emprunté à Moorkens, 2018) cette technologie dès le début de la formation. Ils s'approprieront ainsi un outil d'aide à la traduction qui n'a pas vocation à les remplacer.

Dans cet article, nous abordons plusieurs leviers qui nous paraissent particulièrement pertinents pour atteindre cet objectif. Nous ne prétendons nullement ni détenir les réponses ni savoir avec certitude comment former les traducteurs de demain. Nous avons également conscience que certaines propositions peuvent prêter à débat. Les expériences en la matière sont nombreuses, notamment s'agissant de l'enseignement de la traduction automatique (voir par exemple Massey et Ehrensberger-Dow, 2017;

2. https://www.euatc.org/industry-surveys/item/468-2018-european-language-surveyresults-announced

3. https://csa-research.com/More/Media/Press-Releases/ArticlelD/546/Global-Marketfor-Outsourced-Translation-and-Interpreting-Services-and-Technology-to-Reach-US49-60-Billion-in-2019 
Rossi, 2017; Moorkens, 2018; Faria Pires, 2018 ; Guerberof et Moorkens, 2019; Martikainen, 2019). Les tables rondes et les colloques sur le sujet se multiplient. Notre objectif ici, à partir de notre expérience personnelle de formateur dans le cadre de la formation de master Traduction spécialisée multilingue de l'université de Lille, est de contribuer au débat en proposant des pistes de réflexion. En particulier, nous soulignerons l'importance de présenter la traduction avant tout comme une prestation de service, grâce à des enseignements consacrés à la gestion de projets mais pas uniquement, ou au développement des compétences interpersonnelles (soft skills) par la mise en situation au sein d'agences virtuelles de traduction ou skills labs. Nous proposons également, pour des raisons que nous expliquerons, que les outils de TA soient abordés dès le début de la formation, et non une fois les compétences avancées en traduction acquises. Au-delà de la connaissance, c'est une approche raisonnée prenant en compte les enjeux qui nous semble particulièrement importante: que sait faire la machine? Que ne sait-elle pas faire? Quels sont les risques? Quand avoir recours à ces outils? Quels problèmes déontologiques? Quelle rentabilité? Pour cela, s'il n'est pas obligatoire de comprendre le fonctionnement des moteurs neuronaux dans les moindres détails, il est crucial d'avoir conscience que la performance des outils de TA est en lien direct avec la qualité des données rassemblées en corpus qu'ils exploitent. Les étudiants pourront ainsi déterminer si leur utilisation est pertinente, en évitant de tomber dans le piège de l'illusion que créent parfois ces outils en mettant l'accent sur la fluidité de la langue cible au détriment de la fidélité au texte source (voir l'article de Perrine Schumacher dans ce même numéro, page 113). Et cela sans caricature («piéger» un outil de TA est on ne peut plus facile, mais cela peut être contre-productif) et en formant les étudiants à la déconstruction du discours marketing au profit d'un discours scientifique raisonné, via la découverte de la littérature scientifique sur le sujet, extrêmement prolifique. De façon complémentaire, la maîtrise d'outils informatiques autres, laissant place à la créativité, nous semble également importante pour que le biotraducteur puisse dégager sa plus-value par rapport à la machine. 


\section{Enseigner la traduction d'abord comme une prestation commerciale}

Le référentiel des compétences proposé par le réseau européen des formations de master en traduction de la direction générale de la traduction de la Commission européenne (réseau EMT) ${ }^{4}$ liste, dans sa version mise à jour en 2017, cinq grands types de compétences: (i) langagières (langue et culture), (ii) traductionnelles, (iii) technologiques, (iv) personnelles et interpersonnelles, et ( $v$ ) liées à la prestation de services. Les deux dernières nous semblent à l'heure actuelle particulièrement importantes, dans la mesure où elles ne peuvent être acquises par la machine et représentent des aptitudes spécifiquement "humaines» (il va sans dire que nous n'affirmons pas que les autres compétences sont moins importantes). Au-delà de l'existence d'enseignements consacrés à la gestion de projets qui tendent à se généraliser, former les étudiants à la prestation de services sans jamais leur donner "simplement» un texte à traduire nous semble être une approche à systématiser. Ainsi, chaque document (et non texte) traduit en cours peut s'inscrire dans le cadre d'un projet avec des consignes spécifiques fournies par un client potentiel. Il peut s'agir d'instructions telles que l'utilisation autorisée d'anglicismes ou non, les termes à (ne pas) utiliser obligatoirement, le changement de registre, le guide de style à respecter, la gestion de liens hypertextes, le référencement naturel (SEO), le formatage du document avant livraison, etc. Cette série de services qu'un client peut demander correspond à un ensemble de contraintes que la machine ne sait pas gérer. Tout exercice de traduction, que ce soit dans le cadre des enseignements ou de l'évaluation, devient alors un mini-projet de traduction individuel à gérer avant tout comme une prestation commerciale, avec référence éventuelle à la norme ISO 17100:2015 sur les exigences relatives aux services de traduction.

Afin de développer les compétences interpersonnelles (soft skills), la mise en situation grâce à des dispositifs comme les bureaux de traduction virtuels ou encore skills labs (on pensera également à la Junior entreprise) permet aux étudiants

4. https://ec.europa.eu/info/sites/info/files/emt_competence_fwk_2017_fr_web.pdf 
de mobiliser leurs différentes compétences et les amène à travailler ensemble pour livrer des projets de traduction réalisés collectivement, de la réception à la livraison contre facturation. Chaque étudiant jove alors un rôle au sein du dispositif (gestionnaire de projets, préparateur de ressources et de fichiers, traducteur, réviseur, responsable communication), devenant le maillon d'une chaîne au service des projets de traduction. Ces dispositifs tendent à se répandre (voir le réseau européen INSTB, International Network of Simulated Translation Bureaus) ${ }^{5}$ avec un suivi des progrès des étudiants (Kerremans et al., 2018). Amenés à travailler en autonomie, ces derniers développent leurs compétences interpersonnelles ainsi que leur esprit critique, le tout sous la supervision de traducteurs professionnels en exercice chargés de les accompagner et de les évaluer ${ }^{6}$. Si les compétences liées au transfert linguistique sont naturellement incontournables, elles ne peuvent suffire à la réussite de l'expérience, qui permet à l'étudiant de rassembler les compétences acquises lors de différents enseignements (traduction pratique, recherche documentaire, traduction assistée par ordinateur, révision, gestion de projets, etc.) grâce au décloisonnement. Parmi les choix à effectuer se trouve d'ailleurs celui des outils, donc la question de savoir si la traduction automatique peut ou non être utilisée.

\section{Enseigner la maîtrise des outils de traduction automatique}

Il a longtemps été considéré que l'utilisation des outils de TA exigeait une certaine maturité et une expérience pratique, en partant du principe qu'il faut d'abord savoir déjà très bien traduire avant d'être en mesure de les utiliser à bon escient. Ainsi, la traduction automatique était abordée plutôt en fin de parcours universitaire. Selon nous, cette position

5. Ce réseau rassemble à l'heure actuelle treize universités européennes et vise à développer des partenariats entre les établissements ayant mis en place des dispositifs de simulation (agence virtuelle, bureau de traduction, skills lab). Au-delà d'échanges, il s'agit d'évaluer de façon scientifique les progrès accomplis par les étudiants grâce à ces dispositifs. Pour plus de renseignements: www.instb.eu/

6. Pour un exemple concret du déroulement du dispositif, voir le TSM Skills Lab de l'université de Lille: https://mastertsmlille.wordpress.com/2019/02/18/tsm-skills-lab-2019/ 
n'est plus tenable aujourd'hui: avec la mise à disposition de moteurs génériques et gratuits comme Google Traduction ou DeepL, ou encore de nombreuses applications pour smartphone, les étudiants qui arrivent en formation utilisent déjà les outils de traduction automatique, et ce depuis le collège si l'on en croit les témoignages des enseignants. Il est donc très probable qu'ils les utilisent pour effectuer les traductions demandées par leurs formateurs, quel que soit le type de document. En revanche, ils semblent considérer ces outils comme des outils de "triche». Interrogés sur la question, ce n'est que difficilement qu'ils reconnaissent les utiliser. II y a là selon nous un véritable problème puisque le rôle des enseignants est d'accompagner les futurs traducteurs vers leur vie professionnelle, sachant que les étudiants qui arrivent en formation s'inquiètent également de l'avenir de la profession du fait des avancées technologiques. En passant sous silence, voire en interdisant (sans garantie, d'ailleurs) le recours à la TA, les formateurs ne remplissent pas entièrement leur rôle: les futurs professionnels doivent être formés à la maitrise de ces outils qui feront sans doute partie de leur quotidien. En mettant dès le départ les étudiants en garde contre les risques encourus face à des résultats favorisant la fluidité de la langue cible au détriment de la fidélité au texte source, ce qui tend à créer une illusion de bonne qualité dont les étudiants doivent apprendre à se méfier, le formateur développe l'esprit critique des étudiants vis-à-vis d'une technologie souvent caricaturée par ailleurs ${ }^{7}$. Ainsi, à titre personnel, nous autorisons, y compris en situation d'examen et sans restriction, l'utilisation des outils de TA, avec des résultats tout à fait intéressants: les étudiants débutants qui tentent de

7. Les résultats fournis par les moteurs de TA sont en effet souvent caricaturés, de deux façons différentes. Leurs performances sont régulièrement surévaluées dans la presse généraliste, mais aussi spécialisée, donnant lieu à des titres accrocheurs comme «Google's Neural Machine Translation System: Bridging the Gap between Human and Machine Translation», «Microsoft reaches a historic milestone, using Al to match human performance in translating news from Chinese to English», ou encore "Traducteurs et interprètes: tous chômeurs en 2025?». À l'inverse, des exemples caricaturaux de traductions ridicules circulent massivement sur l'internet tel le fameux "Polish sausage», traduit par "Polissez la saucisse». Ces exemples font rire, mais sont en fait contre-productifs: ils éloignent les étudiants d'une approche raisonnée de la technologie, alors que les exemples choisis sont des phrases averbales, contenant des ambiguïtés lexicales et/ou syntaxiques, ou encore des registres pour lesquels les moteurs de TA sont inopérants (paroles de chanson, poèmes, slogans publicitaires). 
post-éditer des sorties de TA échovent inévitablement, car pour savoir post-éditer correctement un texte, il importe de bien analyser ce que l'on voit, de bien déterminer ce qui doit être vérifié et éventuellement modifié. Ceci est difficile pour les étudiants (voir expérience menée par Yamada, 2019) dans la mesure où certaines des erreurs commises aujourd'hui par les moteurs de TA neuronale se rapprochent de celles que l'on trouve en traduction humaine, d'où une forme d'illusion (voir Deneufbourg, 2019). Par conséquent, seul un œil averti peut accomplir cette tâche, et les étudiants doivent être sensibilisés à cet état de fait. L'utilisation des outils de TA à leurs risques et périls permet de ne plus passer sous silence la technologie, mais de la démystifier: un outil de TA n'est pas une calculatrice et les résultats fournis doivent être systématiquement contrôlés, contrôle qui n'est possible que par un acteur disposant de compétences traductionnelles solides. On pourra d'ailleurs mettre en place des ateliers comparatifs en séparant les étudiants en deux groupes pour la traduction du même texte, l'un proposant une traduction humaine (outillée, mais sans recours à la TA) et l'autre la post-édition d'un texte traduit de façon automatique, afin de bien mettre en lumière ce que la machine sait et ne sait pas faire (voir par exemple Martikainen, 2019). II y a parfois des «surprises»: ainsi on pourrait penser que la machine ne rencontre guère de problèmes s'agissant du report de chiffres (années, pourcentages...) là où le traducteur humain peut faire preuve d'inattention; or ce n'est pas le cas; les omissions par ailleurs ne sont pas rares, par exemple pour la négation ou les adverbes. La confrontation document original/document traduit reste indispensable, mais doit être ciblée.

Si le cloisonnement systématique des enseignements est selon nous à éviter, la présence d'enseignements spécifiques à la traduction automatique, assurés par des professionnels utilisant la TA, semble incontournable. Il est important que les étudiants connaissent l'historique de ces outils et leur fonctionnement au moins de façon sommaire, soient formés aux différents types de post-édition ainsi qu'à la préédition, testent différents moteurs (génériques, spécialisés) afin d'analyser les résultats obtenus et de développer une maîtrise raisonnée. En particulier, les étudiants doivent réfléchir à la question des données utilisées par les moteurs de 
TA pour proposer leurs résultats. De la qualité de celles-ci dépendra bien souvent celle des traductions des moteurs. Par qualité, il importe aussi de comprendre pertinence: comme pour les mémoires de traduction, les données doivent être conformes au projet de traduction concerné. Ainsi, si l'outil de traduction automatique de la direction générale de la traduction de la Commission européenne (eTranslation) fournit de bons résultats avec des textes institutionnels, c'est parce que le corpus sur lequel le moteur est entraîné correspond à ce type de documents. II devient inefficace pour d'autres types de textes. Cette prise de conscience peut être suscitée dans le cadre d'un cours sur les outils de corpus, enseignements qui eux aussi semblent se généraliser (Frérot et Karagouch, 2016). Au-delà de ces questions, les étudiants doivent également être sensibilisés à la manière de gérer les projets de traduction ayant recours à la TA: quels gains de productivité? Comment calculer le tarif par rapport à une traduction $100 \%$ humaine? Quel rapport coût/bénéfice? Les séances de gestion de projets peuvent aborder ces questions, tout comme celles liées à la déontologie: l'utilisation d'outils de TA en ligne n'est pas sans risques en termes de confidentialité. On rappellera à cet égard la mésaventure de cette entreprise norvégienne qui a retrouvé ses données pourtant confidentielles sur l'internet à la disposition de tous en raison du recours à un service de traduction en ligne ${ }^{8}$.

Enfin, d'un point de vue purement linguistique, il est essentiel de faire prendre conscience aux étudiants de l'écart qui sépare langue originale ou traduction humaine d'une part et traduction automatique, post-éditée ou non d'ailleurs, d'autre part. Divers travaux de recherche ont mis au jour les différences linguistiques entre les deux (par exemple Daems et al., 2018; Loock, 2018; Martikainen, 2019). À cet égard, il semble indispensable que les futurs professionnels soient sensibilisés à l'existence de travaux de recherche sur la traduction automatique. Le nombre de publications explose depuis 2017, et si les professionnels ne sont pas des chercheurs, nous en convenons, la lecture de la littérature

8. https://slator.com/technology/translate-com-exposes-highly-sensitive-informationmassive-privacy-breach/ 
scientifique doit être encouragée. En effet, les résultats peuvent tout à fait être exploités dans le cadre d'une pratique professionnelle, en permettant lors de la post-édition la focalisation sur les manquements connus de la machine. Bien souvent, les expériences concluent sur des points forts et des points faibles de tel outil de TA sur la traduction d'un type de document donné. L'évaluation de traductions automatiques, par le biais de métriques ou effectuées par des sujets humains, sur des segments isolés ou des ensembles de traductions réunies en corpus, est un thème de recherche important. La technologie devant encore évolver dans les années à venir, la lecture de littérature scientifique, de plus en plus en accès libre ${ }^{9}$, permet non seulement une approche raisonnée, mais aussi une mise à niveau permanente, avec toujours le souci de maîtriser le recours à la TA dans un cadre professionnel. Il s'agit également, répétons-le, de déconstruire le discours marketing et ses affirmations d'équivalence entre traduction automatique et traduction humaine.

\section{Développer la créativité}

Au-delà des risques déjà évoqués, le recours à la TA peut entraîner la standardisation de la langue, risque existant pour les mémoires de traduction. Si les nouvelles traductions s'inspirent d'anciennes, quelle part reste-t-il pour la créativité? II faut donc inviter les étudiants à développer celle-ci en utilisant des outils requérant une démarche active et non passive: plutôt que de voir une traduction s'afficher sur l'écran dont il peut être difficile de s'éloigner une fois lue, il pourra leur être conseillé de consulter des données réunies en corpus afin d'observer différentes possibilités linguistiques et non une proposition unique. Cela peut se faire au sein des mémoires de traduction grâce à des fonctionnalités de recherche dans le logiciel de TAO, mais aussi en exploitant des corpus de données monolingues consultables en ligne ou hors-ligne (voir Loock 2016a/b à ce sujet). La popularité des outils de corpus aujourd'hui auprès des traducteurs, comme

9. On citera par exemple les actes des différents Machine Translation Summits, systématiquement mis en ligne. Ainsi, pour 2019, voir https://www.mtsummit2019.com/ 
en témoigne le nombre de formations sur le sujet après toute une période que l'on pourra qualifier de rendez-vous manqué, s'explique selon nous justement par le souhait des traducteurs d'effectuer leurs propres choix, et d'exprimer ainsi leur créativité. Par opposition, les traductions fournies par la TA, ainsi que les mémoires de traduction peuvent venir la brider et in fine standardiser la langue des textes traduits.

\section{Conclusion}

A partir de notre expérience personnelle de formateur, nous avons souhaité proposer ici quelques réflexions sur un enjeu qui nous semble majeur pour les formations en traduction: comment préparer les futurs professionnels à des métiers qui rencontrent de grands bouleversements technologiques avec l'arrivée de la traduction automatique neuronale? Nous avons abordé différentes perspectives et différents leviers permettant une approche raisonnée amenant les étudiants à prendre conscience de leur plus-value par rapport à la machine.

II s'agit là d'une simple contribution au débat qui bat son plein à l'heure actuelle sur la façon de former les futurs traducteurs, comme le montrent ce numéro de la revue Traduire, les réflexions menées dans le cadre du réseau européen EMT ou encore de l'Association française des formations universitaires aux métiers de la traduction ( $A F F \cup M T$ ), qui organise un colloque international sur ce sujet en mai $2020^{10}$. Nous constatons d'ailleurs à cet égard une véritable convergence des intérêts des mondes professionnel et universitaire, qui ne peut que déboucher sur une collaboration renforcée à l'avenir.

Nous souhaitons chaleureusement remercier Nicolas Froeliger pour sa relecture d'une première version.

rudy.loock@univ-lille.fr https://pro.univ-lille.fr/rudy-loock/

10. https://affumt2020.sciencesconf.org/ 
Rudy Loock est professeur des universités en linguistique anglaise et en traductologie au sein de I'UFR Langues étrangères appliquées de I'université de Lille. Responsable du parcours de master Traduction spécialisée multilingue, il est aussi membre de I'UMR Savoirs, Textes, Langage du CNRS. II a publié en 2016 La Traductologie de corpus aux Presses universitaires du Septentrion. Il est depuis 2018 le président de l'Association française des formations universitaires aux métiers de la traduction (AFFUMT).

SOURCES CitÉES

DAEMS Joke et al., «Translationese and post-editese: How comparable is comparable quality?», in Linguistica Antverpiensia, New Series: Themes in Translation Studies, 16, 2017, p. 89-103.

DENEUFBOURG Guillaume, «Post-édition de traduction automatique : se méfier des apparences », 2019, www.ata-divisions.org/FLD/index.php/tag/guillaumedeneufbourg, consulté le 21 octobre 2019.

FARIA PIRES Loïc de, "Intégration de la traduction automatique neuronale à la formation universitaire des futurs traducteurs: pistes d'exploration », in Myriades, 4, 2018, p. 53-65.

FRÉROT Cécile et KARAGOUCH Lionel, «Outils d'aide à la traduction et formation de traducteurs: vers une adéquation des contenus pédagogiques avec la réalité technologique des traducteurs », in ILCEA, 27, 2016, http://journals.openedition.org/ilcea/3849, consulté le 21 octobre 2019.

FROELIGER Nicolas, Les Noces de l'analogique et du numérique. De la traduction pragmatique, Paris, Les Belles Lettres, 2013.

GUERBEROF ARENAS Ana et MOORKENS Joss, «Machine translation and postediting training as part of a master's programme», in Journal of Specialised Translation, 31, 2019, p. 217-238.

KERREMANS Koen et al., «Assessing interpersonal skills in translator training: the cases of INSTB», 2018, www.instb.eu/publications/, consulté le 20 août 2019.

LOOCK Rudy, «Traduction automatique et usage linguistique: une analyse de traductions anglais-français réunies en corpus », in Meta, 3, 2018, p. 785-805.

LOOCK Rudy, La Traductologie de corpus, Villeneuve-d'Ascq, Presses universitaires du Septentrion, 2016a.

LOOCK Rudy, «L'utilisation des corpus électroniques chez le traducteur professionnel: quand? comment? pour quoi faire? », in ILCEA, 27, 2016b, https://journals.openedition.org/ilcea/3835, consulté le 21 octobre 2019. 
MARTIKAINEN Hanna, «Post-Editing Neural MT in Medical LSP: LexicoGrammatical Patterns and Distortion in the Communication of Specialized Knowledge», in Informatics, 6, 2019, https://www.mdpi.com/2227-9709/6/3/26/ htm, consulté le 21 octobre 2019.

MASSEY Gary et EHRENSBERGER-DOW Maureen, «Machine learning: Implications for translator education», in Lebende Sprachen, 62 (2), 2017, p. 300-312.

MOORKENS Joss, «What to expect from Neural Machine Translation: a practical in-class translation evaluation exercise», in The Interpreter and Translator Trainer, 12 (4), 2018, p. 375-387.

ROSSI Caroline, «Introducing statistical machine translation in translator training: From uses and perceptions to course design and back again», in Revista Tradumàtica. Tecnologies de la Traducció, 15, 2017, p. 48-62.

YAMADA Masaru, «The impact of Google Neural Machine Translation on postediting by student translators», in Journal of Specialised Translation, 31, 2019, p. 87-106. 